\title{
Acute Reversible Motor Predominant Polyradiculoneuropathy among Thai Prisoners after the Outbreak of H3N2 Influenza: A Case Series
}

\author{
Phichamon Khanittanuphong, M.D. ${ }^{1}$, Thanyalak Amornpojnimman, M.D. ${ }^{2}$, \\ Sirichai Cheewatanakornkul, M.D. ${ }^{3}$, Watchara Viratyaporn, M.D. ${ }^{4}$, \\ Ittipon Preechawettayakul, M.D. ${ }^{5}$, Chorfa Buntong, R.N. ${ }^{6}$, Pat Korathanakhun, M.D. ${ }^{7}$, \\ Erin Kathleen O’Ferrall, M.D., M.Sc. ${ }^{7,8}$
}

${ }^{1}$ Department of Rehabilitation Medicine, Faculty of Medicine, Prince of Songkla University, Hat Yai, Songkhla 90110, Thailand. ${ }^{2}$ Neurology Unit, Department of Internal Medicine, Faculty of Medicine, Prince of Songkla University, Hat Yai, Songkhla 90110, Thailand.

${ }^{3}$ Cadiology Unit, Department of Internal Medicine, Faculty of Medicine, Prince of Songkla University, Hat Yai, Songkhla 90110, Thailand.

${ }^{4}$ Department of Internal Medicine, Hatyai Hospital, Hat Yai, Songkhla 90110, Thailand.

${ }^{5}$ Department of Internal Medicine, Na-Thawi Hospital, Na-Thawi, Songkhla 90160, Thailand.

${ }^{6}$ Nursing Unit, Na-Thawi District Prison, Na-Thawi, Songkhla 90160, Thailand.

${ }^{7}$ Departments of Neurology and Neurosurgery, Faculty of Medicine, McGill University, Montréal Neurological Institute and Hospital, Montréal, Québec H3A 2B4, Canada.

${ }^{8}$ Department of Pathology, Faculty of Medicine, McGill University, Montréal Neurological Institute and Hospital, Montréal,

Québec H3A 2B4, Canada.

Received 23 April $2020 \bullet$ Revised 18 June 2020 • Accepted 23 June $2020 \bullet$ Published online 2 November 2020

\begin{abstract}
:
Objective: This study aims to describe the clinical, laboratory and electrophysiological findings of the prisoners who developed acute reversible motor predominant polyradiculoneuropathy after the outbreak of H3N2 influenza.

Material and Methods: Among H3N2 infected Thai prisoners, all patients with acute flaccid weakness were included in this retrospective case series. We analyzed the results of electromyography (EMG), cerebrospinal fluid analysis, and serum levels of antiganglioside antibodies, folate, thiamine, and vitamin B12.

Contact: Pat Korathanakhun, M.D.

Division of Neurology, Department of Internal Medicine, Faculty of Medicine,

Prince of Songkla University, Hat Yai, Songkhla 90110, Thailand.

E-mail: patosk120@gmail.com

Prince of Songkla University. All rights reserved.

This is an open access article under the CC BY-NC-ND license

(http://www.jhsmr.org/index.php/jhsmr/about/editorialPolicies\#openAccessPolicy).
\end{abstract}


Results: Among 262 H3N2 influenza patients, motor predominant polyradiculoneuropathy predominantly affecting the lower limbs developed in 10 patients. EMG revealed significantly decreased compound muscle action potentials with preserved distal latencies and conduction velocities in the peroneal nerves. Early fibrillation potentials were demonstrated on EMG performed 12-14 days post-onset of symptoms. The cerebrospinal fluid study showed normal profiles. All laboratory results appeared normal except for the significantly low mean \pm standard deviation serum folate level of $1.80 \pm$ 0.78 [reference value 3.89-26.80 nanograms per milliliter $(\mathrm{ng} / \mathrm{mL})$ ] as compared to the mean serum folate level in the normal Thai population of $10.80 \mathrm{ng} / \mathrm{mL}$ ( $p$-value $<0.001,95 \%$ confidential interval -9.55 to -8.45 ). All patients were treated with 15 milligram per day of folic acid and all patients improved clinically.

Conclusion: Although the relationship between low plasma folate level and polyradiculoneuropathy is unclear, a superimposed folate deficiency contributing to the pathophysiology is possible.

Keywords: folate deficiency, H3N2, influenza, polyradiculoneuropathy

\section{Introduction}

Acute flaccid paralysis is characterized by rapid onset of weakness progressing to maximum severity within several days to weeks. The differential diagnosis of acute flaccid paralysis is broad and includes diseases of the nerve, neuromuscular junction and motor neuron. For anterior horn cell disease, apart from the most common contagious disease, acute poliomyelitis which selectively affected the motor neuron, post vaccinated myelitis and neurotropic virus infection should also be kept in mind. In the peripheral nerve category, various conditions are listed including Guillain-Barré syndrome (GBS), acute toxic neuropathies (heavy metals), arthropod bites and neuropathies of infectious diseases (diphtheria and lyme disease). Neuromuscular junction disorders such as myasthenia gravis, botulism, tick bite paralysis and organophosphate poisoning are great mimickers. Lastly, myopathy, for instance hypokalemic periodic paralysis and post viral myositis, can also cause flaccid weakness. ${ }^{1}$

In July 2017, an outbreak of H3N2 influenza among 262 prisoners in the Na-Thawi District Prison of Thailand was identified. Shortly after the onset of this outbreak, ten prisoners developed acute progressive, symmetrical, distally predominant numbness and weakness mimicking Guillain-Barré syndrome. This study aims to describe the clinical, electromyography (EMG) and laboratory characteristics of polyradiculoneuropathy in association with low serum folate levels.

\section{Material and Methods \\ Study design}

A case series study was conducted in the Na-Thawi District Prison located in Songkla Province, Thailand, during the H3N2 influenza outbreak. Out of 400 prisoners, 262 prisoners were confirmed infected with H3N2 in July 2017. The H3N2 influenza infection was detected by immunofixation assay of a nasopharyngeal swab sample and was confirmed by the polymerase chain reaction method.

\section{Inclusion and exclusion criteria}

The patients enrolled in this study met the following criteria: (1) developed acute flaccid weakness during the influenza outbreak in the prison and (2) consented for the use of all investigational data required for this study. Patients with diabetes mellitus or known neuromuscular disease 
were excluded. The study was approved by the ethical committee of the Faculty of Medicine, Prince of Songkla University. Informed consent was completed for all patients.

\section{Terminology}

To avoid a misunderstanding between the duration and the onset time of fever and weakness, specific terms were defined as follows: (1) fever duration was the time between the date of first observed fever to the date of fever resolution (days) and (2) time to weakness onset was the duration between the date of fever onset to the date of first observed weakness (days).

\section{Data collection}

The study protocol included medical history taking and physical examination by neurologists. We collected baseline characteristics and prodromal symptoms prior to the onset of weakness and clinical signs.

Blood tests to exclude other confounding medical conditions included: complete blood count, liver and renal function test, electrolytes, thyroid stimulating hormone, thiamine, vitamin B12, folate, and serum anti-gangliosides. Cerebrospinal fluid (CSF) analysis was performed. The whole blood samples were preserved with ethylenediaminetetraacetic acid and stored according to the lightprotection protocol for the measurement of the thiamine level by high-performance liquid chromatography. The plasma folate and vitamin B12 levels were analyzed by the electrochemiluminescence immunoassay analyzer. The serum anti-gangliosides panel (GM1, GM2, GM3, GD1a, GD1b, GT1b, and GQ1b) was examined by the western blot method. Needle EMG and nerve conduction studies (NCS) were performed by a board-certified electromyographer using the Dantec ${ }^{\mathrm{TM}}$ Keypoint $^{\circledR}$ portable EMG system, Natus Medical Incorporated Company, The United Stated of America. An antidromic sensory NCS was tested at the right median nerve, bilateral ulnar nerves, and bilateral sural nerves. The motor NCS was performed at the right median nerve (recording at the abductor pollicis brevis muscle), bilateral ulnar nerve (recording at the abductor digiti minimi muscle), bilateral peroneal nerve (recording at the extensor digitorum brevis muscle), and right tibial nerve (recording at the abductor hallucis muscle). The EMG needle electrode was inserted in the right tibialis anterior muscle and right flexor carpi ulnaris muscle. Clinical follow-up visits were performed monthly.

\section{Data analysis}

Discrete data are described by number and percentage and continuous data are reported as mean \pm standard deviation (S.D.) or median [interquartile (IQR) 1 and 3]. In order to examine the statistical difference, the mean serum folate in our population was analyzed by the one sample $z$-test method with an alpha of 0.05 compared to the mean serum folate of the normal Thai population in a study by Areekul et al. ${ }^{2}$ Statistical significance was determined if $p$-value $<0.050$.

\section{Results}

\section{Demographic data and clinical features}

There are 850 female prisoners and 2240 male prisoners in this prison. The H3N2 outbreak occurred only in the male prison area. Among 262 male prisoners with confirmed influenza infection, ten male patients with acute flaccid weakness were enrolled in the study. None of the patients with weakness had comorbid diabetes mellitus or any known neuromuscular disease. The mean \pm S.D. age was $27.2 \pm 4.6$ years old and the mean \pm S.D. duration of imprisonment was $12.2 \pm 4.7$ months.

All patients presented with fever, numbness, and symmetrical weakness. The mean \pm S.D. fever duration was $2.4 \pm 2.0$ days while the mean \pm S.D. time to onset of weakness was $7.5 \pm 4.3$ days which indicates a 5 day 
delay between fever and onset of weakness. On the date of evaluation, none of the patients had fever or signs of active infection. Proximal leg weakness and distal leg weakness was observed in $100.0 \%$ and $90.0 \%$ of the patients, respectively, which led to the inability to walk in $40.0 \%$. In contrast, signs of weakness in the proximal arm, distal arm, and neck muscles were observed in only $50.0 \%, 40.0 \%$, and $20.0 \%$ of the patients, respectively. Hyporeflexia was observed in all patients and progressed to the absence of deep tendon reflex in $40.0 \%$. Lengthdependent pinprick sensation impairment was found in $80.0 \%$ while proprioceptive and vibratory sensation defects were detected in only $10.0 \%$. None of the patients experienced pain in lower limbs or dysfunction of bowel and bladder. Neither ophthalmoplegia nor facial diplegia was found.

None of the patients developed clinical signs of thiamine deficiency (wet beriberi) (i.e. neck vein engorgement, lung crepitation, heart murmur, shifting of apical beat, or leg edema). The mean \pm S.D. systolic blood pressure was $131.7 \pm 28.5 \mathrm{mmHg}$, mean \pm S.D. diastolic blood pressure was $79.0 \pm 18.1$ millimeter mercury $(\mathrm{mmHg})$, and mean \pm S.D. pulse rate was $97.1 \pm 26.2$ beats per minute with a regular rhythm. The mean \pm S.D. body weight was $59.8 \pm$ 5.9 kilograms $(\mathrm{kg})$ with a mean \pm S.D. body mass index (BMI) of $21.3 \pm 1.4$ kilograms per square meter. None of the patients had any skin signs that would suggest arsenic or lead poisoning.

\section{Nerve conduction studies and electromyo- graphic findings}

The NCS indicated lower limb-predominant axonal polyradiculoneuropathy. The motor NCS revealed decreased compound muscle action potential amplitudes of the peroneal nerves bilaterally with preserved distal latencies and conduction velocities in 9 patients and abnormal F-wave latency in 5 patients while the motor NCS in the other nerves were only slightly affected. The sensory NCS elicited mildly decreased sensory nerve action potential (SNAP) amplitudes below the lower limit of normal in 6 patients (median nerves, ulnar nerves and sural nerves). The sensory NCS in the other 4 patients showed the difference in the SNAP amplitudes of the sural nerves from side to side exceeded $50.0 \%$ which indicated subtle abnormalities. One patient (patient 7) revealed preserved despite the presence of clinically sensory deficit which may indicate pathology proximal to the dorsal root ganglion. The needle EMG demonstrated fibrillation potentials, positive sharp waves, and reduced recruitment with polyphasic motor unit action potentials consistent with acute neurogenic changes in right tibialis anterior and flexor carpi radialis muscles (Table 1). Overall, the NCS and EMG were compatible with a sensory and motor axonal polyradiculoneuropathy in all 10 patients.

\section{Laboratory investigations and a comparison} of the mean serum folate level with the normal level among the Thai population

Several investigations were performed to identify the cause of axonal polyneuropathy. The CSF analyses at the end of the second week did not show cytoalbuminologic dissociation. Serum ganglioside antibodies (GM1, GM2, GM3, GD1a, GD1b, GT1b, and GQ1b) were negative in all patients. The other laboratory results appeared normal except the mean \pm S.D. serum folate level of $1.80 \pm 0.78$ nanograms per milliliter $(\mathrm{ng} / \mathrm{mL})$ which was lower than the reference value (3.89-26.80 $\mathrm{ng} / \mathrm{mL})$ (Table 2). The mean serum folate in our population of ten patients $(1.80 \mathrm{ng} / \mathrm{mL})$ compared to the normal serum folate level in the normal Thai population $(10.80 \mathrm{ng} / \mathrm{mL})$ from a study by Areekul et $\mathrm{al}^{2}{ }^{2}$ revealed a mean difference value of $9.002(\mathrm{z}=$ -36.697, p-value $<0.001,95 \%$ confidential interval -9.55 to -8.45). In addition, a review of the nutritional content served to the prisoners in the previous 3 month period revealed a lack of folate-rich foods, in particular fruits and vegetables, which potentially contributed to folate storage depletion. 


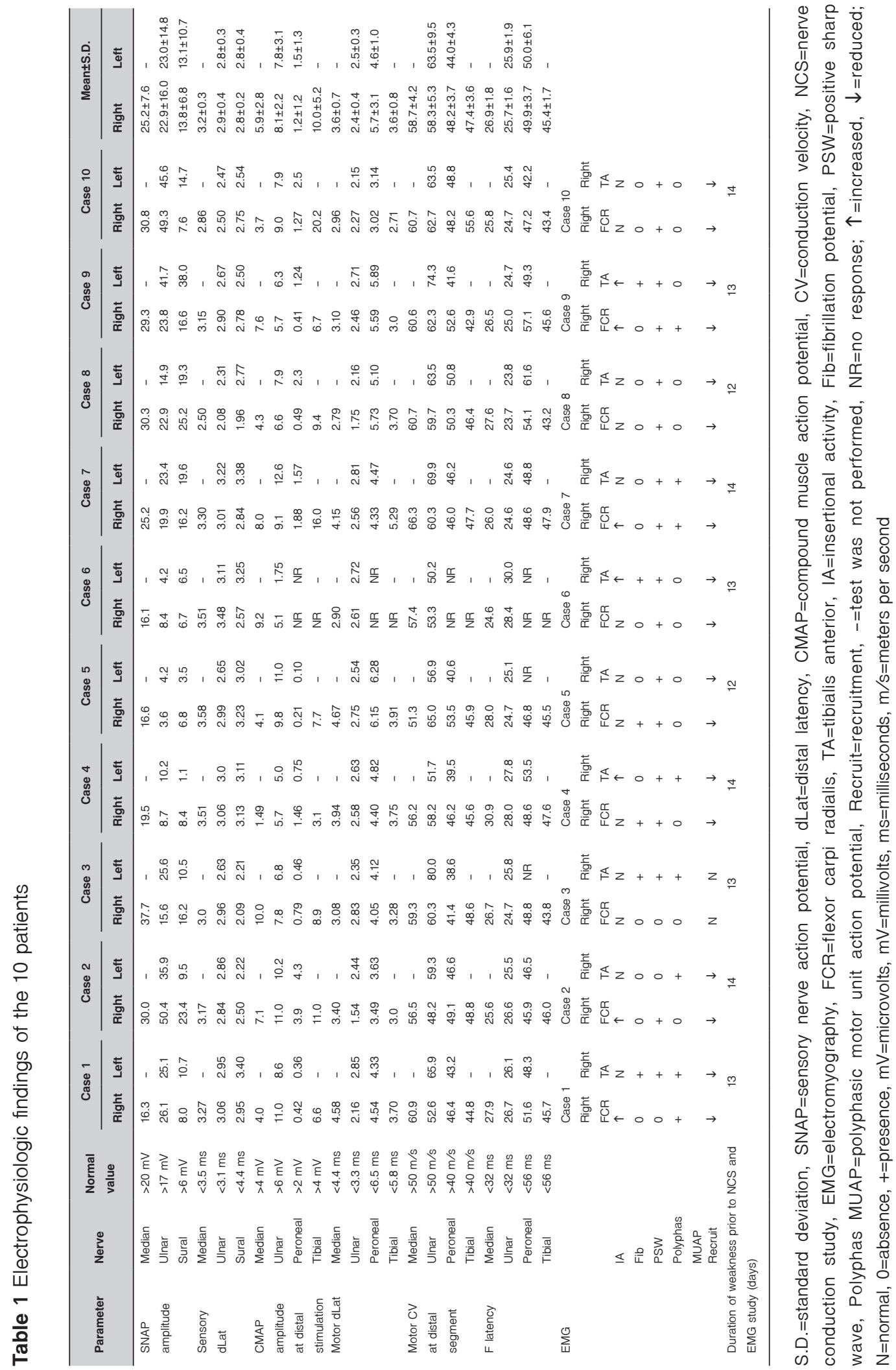




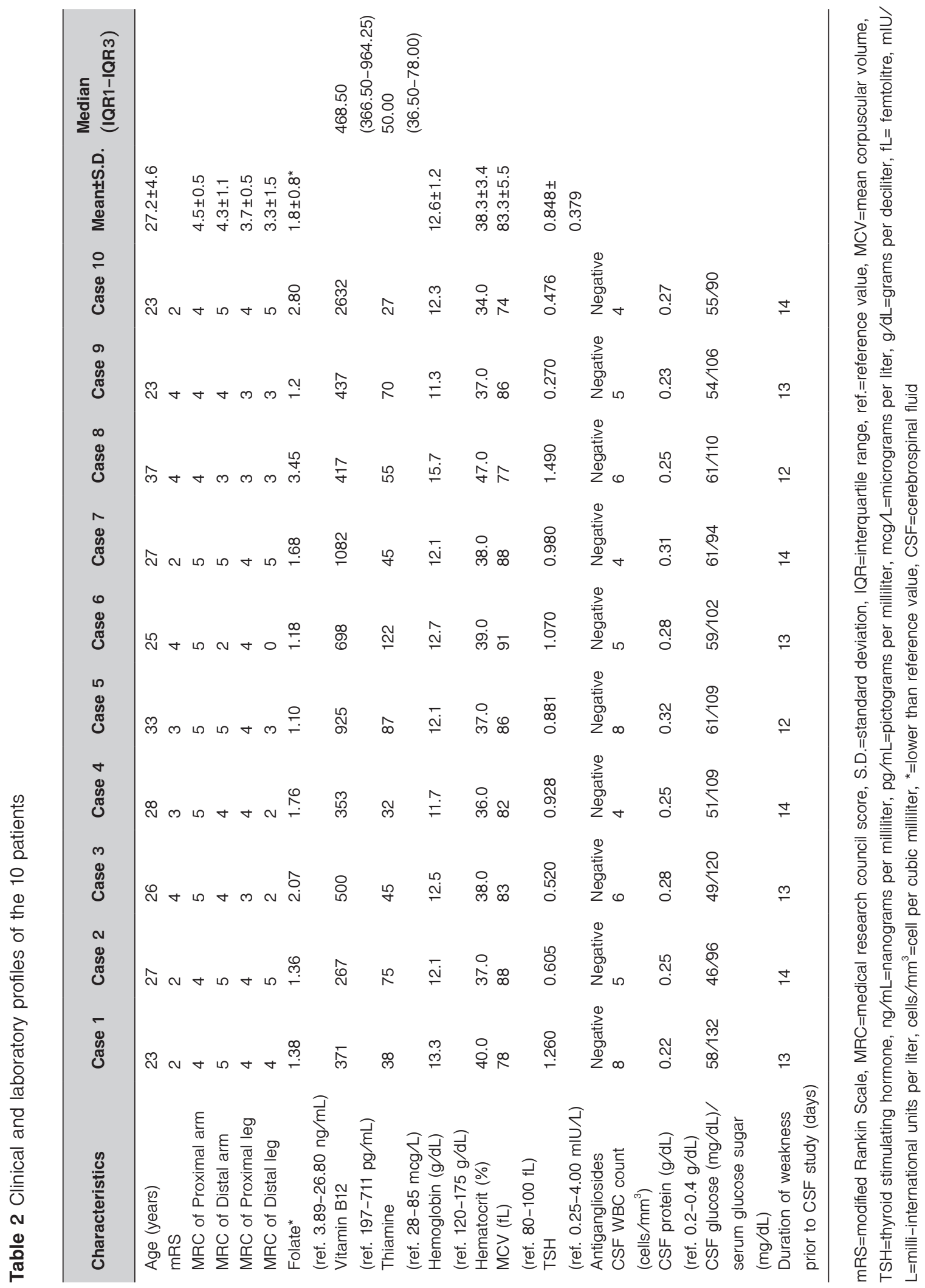


Finally, as the extremely low plasma folate level was the only abnormality detected supplementary folic acid at 15 milligrams per day orally was prescribed. After treating with folic acid for 2 months, the symptoms of weakness gradually improved in all patients and returned to normal function by the end of the third month.

\section{Discussion}

This retrospective case series describes 10 patients who developed acute motor predominant polyradiculoneuropathy shortly after H3N2 influenza infection during an outbreak among Thai prisoners. The acute onset, rapid progression, motor predominance and areflexia and NCS findings were suggestive of GBS. Although GBS is known to be associated with influenza infections, several elements in our series are atypical for GBS.

First, the typical CSF profile in GBS is a high CSF protein level with normal CSF white blood cell (WBC) count, also known as cytoalbuminologic dissociation. The CSF profiles of the patients in our series showed absolutely normal CSF protein levels with normal CSF WBC in the second week of weakness, a finding that argues against GBS. Previous studies have demonstrated elevated CSF protein with albuminocytological dissociation present in $73.2 \%$ buy the second week of symptoms. ${ }^{3}$

Second, since GBS is an immune-mediated inflammatory process triggered by an immunogenic antigen from certain infections, this molecular mimicry of an immunogenic process classically takes 2-3 weeks to be activated and it can extend to 5-9 weeks. ${ }^{4-6}$ However, the lag time between the influenza infection and the onset of weakness in this study was approximately 1 week which was too early for typical post-infectious GBS.

Third, worldwide reports on the annual incidence of post-influenza GBS was 1.72 cases per 100,000 influenza infection. ${ }^{7}$ However, the rate of post-H3N2 polyradiculo- neuropathy in this study [10 cases in 262 influenza (estimated 3,816.8 cases per 100,000 influenza)] significantly exceeds the reported post-influenza GBS incidence.

Fourth, the EMG showed early fibrillations in distal muscles on the $14^{\text {th }}$ day post-onset of weakness. On the basis of neurophysiology, the fibrillation potential and positive sharp wave are typically detected at the third week after denervation. ${ }^{7,8}$ The discordance between the abnormal chronological change in the EMG finding and the duration of weakness does not support the diagnosis of GBS.

The only positive finding from the laboratory investigations was the extremely low serum folate level beyond the lower normal value. This finding raises the question if a low serum folate level is endemic among the Thai population. Although the blood test was not performed in a normal control population, we compared the mean serum folate in our patients to the normal range among the Thai population in a previous study. ${ }^{2}$ The results showed a significant difference in the mean serum folate levels between the two groups which confirmed the extremely low mean serum folate to the level of deficiency in our study. Furthermore, a nutritional review of the diet served to the prisoners confirmed a lack of folate-rich fruits and vegetables.

Regarding the clinical feature, our study showed predominant motor involvement that differed from the sensory predominant polyneuropathy in the previous study. However, the mechanism of folate deficiency and neuropathy development remains unclear and is a further research area.

Although the classic case report and case series study of a folate-deficiency polyneuropathy exhibiting a chronic progressive clinical course among non-alcoholic patients with longstanding malnutrition and among patients with a combination of gastrointestinal pathology with chronic nutritional deficiency, one study by Koike et al. elucidated an acute onset of folate deficiency poly- 
neuropathy. ${ }^{9-11}$ In this study with an acute presentation, we hypothesized that the acute fever in response to the influenza increased folate consumption leading to acute depletion of folate storage.

Folate receptor is a cell surface glycoprotein particle which can be classified into 3 isoforms: alpha, beta, and gamma. In addition, folate receptors are known to be highly expressed on activated macrophages. In the influenza infection model, macrophage plays a major role in the immune response against influenza virus. ${ }^{12}$ Although there is no direct evidence of the folate receptor function in influenza infection model, the autoimmune disease model (rheumatoid arthritis, inflammatory bowel disease, etc.) revealed the upregulation of the beta-folate receptor with significantly increasing function in response to inflammation. ${ }^{13,14}$ Therefore, the increased folate requirement at the cellular level in response to fever and inflammation potentially depletes the serum folate level. ${ }^{15}$ The isolated folate deficiency in our patients with normal body weights and BMI, normal levels of vitamin B12 and thiamine as well as the normal basic laboratory investigations further supports the hypothesis of a selective folate deficiency precipitated by fever rather than a general state of malnutrition.

As this study was conducted under limited circumstances in the prison, some limitations need to be mentioned. First for ethical reasons, the investigators were not allowed to obtain blood samples from the healthy prisoners or the influenza-infected prisoners who did not develop weakness. Therefore, there was no control group to compare in this study. In order to correct this point, we examined the statistical significance of the mean difference between the serum folate level in our study and those in the normal Thai population from a previous study. ${ }^{2}$ Another drawback is the small number of subjects enrolled into the study; however, this study was an investigation of an outbreak in a certain environment where we could not expand the number of subjects. Lastly, due to the prison escape prevention policy, once the patients had recovered (returned to baseline strength), transfer for a follow-up electrophysiological study in the hospital and blood test for serum folate level were not permitted. Therefore, only the clinical follow-up (neurological examination) was performed at the prison.

\section{Conclusion}

We found a series of acute motor predominant polyradiculoneuropathy following the H3N2 influenza infection among the prisoners and the low serum folate level beyond the normal value. In addition, the clinical course differed from the classic folate deficiency polyneuropathy. The relationship between folate deficiency and polyradiculoneuropathy in our cohort remains unclear.

\section{Acknowledgement}

We gratefully thank the collaboration from the Bureau of Epidemiology, Department of Disease Control, Ministry of Public Health, and the disease investigation team comprised of Dr. Suwat Wiriyapongsukit, Dr. Don Intanon, Dr. Papassorn Wankhan, Dr. Sasithorn Saengprateep, Dr. Tabtim Chongsuvivatwong, Dr. Songchai Kittipanprayoo, Dr. Kiatsak Rajborirug, and Mrs. Atchara Boonmeeprakob. The English editing of this manuscript by Mr. Geoffrey Cox is also appreciated.

\section{Funding sources}

This study was supported by Songklanagarind Hospital, Faculty of Medicine, Prince of Songkla University, Thailand.

\section{Conflict of interest}

The authors declared no conflict of interest in this study. 


\section{References}

1. Marx A, Glass JD, Sutter RW. Differential diagnosis of acute flaccid paralysis and its role in poliomyelitis surveillance. Epidemiol Rev 2000;22:298-316.

2. Areekul S, Cheeramakara C, Churdchu K, Pongpaew P, Suwanbun N. Serum folate and vitamin B12 levels in chronic alcoholics. Siriraj Hosp Gaz 1993;45:14-8.

3. Zeng Y, Liu Y, Xie Y, Liang J, Xiao Z, Lu Z. Clinical Features and the validation of the brighton criteria in Guillain-Barré syndrome: retrospective analysis of 72 hospitalized patients in three years. Eur Neurol 2019;1:1-8.

4. Willison HJ, Goodyear CS. Glycolipid antigens and autoantibodies in autoimmune neuropathies. Trends Immunol 2013;34:453-9.

5. Jacobs BC, Rothbarth $\mathrm{PH}$, van der Meché FG, Herbrink $P$, Schmitz PI, de Klerk MA, et al. The spectrum of antecedent infections in Guillain-Barré syndrome: a case-control study. Neurology 1998;51:1110-5.

6. Paploski IA, Prates AP, Cardoso CW, Kikuti M, Silva MM Waller LA, et al. Time lags between exanthematous illness attributed to Zika Virus, Guillain-Barré syndrome, and microcephaly, Salvador, Brazil. Emerg Infect Dis 2016;22:143844.

7. Kwong JC, Vasa PP, Campitelli MA, Hawken S, Wilson K, Rosella LC, et al. Risk of Guillain-Barré syndrome after seasonal influenza vaccination and influenza health-care encounters: a self-controlled study. Lancet Infect Dis 2013; 13:769-76.

8. Dumitru D, King JC. Fibrillation potential amplitude after denervation. Am J Phys Med Rehabil 1998;77:483-9.

9. Koike H, Takahashi M, Ohyama K, Hashimoto R, Kawagashira $\mathrm{Y}$, lijima $\mathrm{M}$, et al. Clinicopathologic features of folate-deficiency neuropathy. Neurology 2015;84:1026-33.

10. Botez MI, Peyronnard JM, Bachevalier J, Charron L. Polyneuropathy and folate deficiency. Arch Neurol 1978;35:581-4.

11. Koike H, Hama T, Kawagashira $Y$, Hashimoto R, Tomita M, lijima $\mathrm{M}$, et al. The significance of folate deficiency in alcoholic and nutritional neuropathies: analysis of a case. Nutrition 2012;28:821-4.

12. Kreijtz JH, Fouchier RA, Rimmelzwaan GF. Immune responses to influenza virus infection. Virus Res 2011;162:19-30.

13. Xia W, Hilgenbrink AR, Matteson EL, Lockwood MB, Cheng JX, Low PS. A functional folate receptor is induced during macrophage activation and can be used to target drugs to activated macrophages. Blood 2009;113:438-46.

14. Yi YS. Folate receptor-targeted diagnostics and therapeutics for inflammatory diseases. Immune Netw 2016;16:33743.

15. Osifo BO, Lukanmbi FA, Familusi JB. Increase of body temperature and folic acid metabolism. Acta Vitaminol Enzymol 1981;3:177-81 